Reselr

\title{
RasGRF1 regulates the \\ hypothalamic-pituitary-adrenal axis specifically in early-adolescent female mice
}

\author{
Belkis Gizem Uzturk', Shan-xue Jin², Beverly Rubin ${ }^{1,3}$, \\ Christopher Bartolome ${ }^{1}$ and Larry A Feig ${ }^{1,2}$
}

${ }^{1}$ Sackler School of Graduate Biomedical Sciences, Departments of ${ }^{2}$ Developmental, Molecular and Chemical Biology, and ${ }^{3}$ Integrated Physiology and Pathobiology, Tufts University School of Medicine, Boston, Massachusetts 02111, USA
Correspondence should be addressed to $L$ A Feig

Email

larry.feig@tufts.edu

\begin{abstract}
Dysregulation of the hypothalamic-pituitary-adrenal (HPA) axis has been implicated in the induction and prolongation of a variety of psychiatric disorders. As such, much effort has been made to understand the molecular mechanisms involved in its control. However, the vast majority of the studies on the HPA axis have used adult animals, and among these the majority has used males. Here we show that in knockout mice lacking the guanine
\end{abstract} Key Words

- RasGRF1

- HPA axis

- adolescence

- female
Journal of Endocrinology (2015) 227, 1-12

\section{Introduction}

The hypothalamic-pituitary-adrenal (HPA) axis plays a vital role in restoring homeostasis following environmental challenge. Physical or psychological stress results in cortisol production in humans or corticosterone (CORT) in rodents that alters a variety of adaptive animal responses. When stress is sensed, PVN cells of the hypothalamus release corticotrophin-releasing hormone
(CRH) (and AVP), which releases adrenocorticotropic hormone (ACTH) from the pituitary into the circulation. ACTH promotes the release of CORT from the adrenal glands, which interacts with its receptors present throughout the body that carry out the stress response. To limit HPA axis responses, critical short- and long-term negative feedback systems exist that are driven, at least in part,

Published by Bioscientifica Ltd. 
by circulating CORT that changes gene expressions in key brain regions. These include direct effects on hypothalamic PVN cells and indirect effects through the hippocampus and the medial prefrontal cortex (mPFC) that suppress $\mathrm{CRH}$ release by activating inhibitory neurons that contact PVN cells. In contrast, positive feedback occurs via the amygdala (Jankord \& Herman 2008). In humans, HPA hyper-reactivity is linked to vulnerability to stress and heightened risk for depression and anxiety disorders (O'Keane et al. 2012). Moreover, excessive suppression of the HPA axis is associated with the development of post-traumatic stress disorder (PTSD; Handwerger 2009).

A key aspect of HPA axis response to stress is that it habituates after multiple similar exposures, which leads to decreased CORT response (Grissom \& Bhatnagar 2009). It is a key mechanism for preventing damaging effects of continuous high levels of glucocorticoids, and it is possible that the transition to stress-induced pathology may involve a breakdown in habituation. The rate of habituation is inversely proportional to severity of the stressor (Grissom \& Bhatnagar 2009). Moreover, the rate of habituation can be regulated by the environment, such as changing context. Habituation is not a reversion to normal because it involves long-term changes to CNS circuits and is reversible in time (Grissom \& Bhatnagar 2009). The neural mechanisms involved in habituation appear to involve the sensory cortex and the limbic system, including the mPFC (Weinberg et al. 2010), basal amygdala (Grissom \& Bhatnagar 2011), and paraventricular thalamus (Bhatnagar et al. 2002), but the molecular mechanisms in each tissue and whether this is the case in adolescent females remain obscure.

The majority of preclinical studies designed to understand the regulation of the HPA axis response to stress have involved adult animals and in most cases have used males. This is a weakness, because treatments designed to overcome psychiatric disorders that involve dysregulation of the HPA axis based on these studies may not be appropriate for adolescent females if the fundamental mechanisms of the HPA axis regulation are different in these populations. Many differences have already been observed in the way both female (McCormick et al. 2008, 2010, Goel et al. 2014) and adolescent populations (Romeo et al. 2006, Andersen \& Teicher 2008, Romeo 2010, Barha et al. 2011, Burke \& Miczek 2014, Hall \& Romeo 2014) respond to stress and to treatments designed for psychiatric disorders thought to be associated with defective HPA axis function, but differences in the biochemical pathways in key brain regions involved in the control of the HPA axis response to stress and habitation to it specifically in adolescent females are poorly understood.

p140-RasGRF1(GRF1) and p130-RasGRF2(GRF2) constitute a family of calcium-activated exchange factors for both Ras and Rac GTPases that are expressed in CNS neurons but not glia (Feig 2011). Both proteins mediate the action of NMDA-type glutamate receptors, but in opposite ways in the CA1 hippocampus. GRF2 mediates long-term potentiation (LTP), while GRF1 mediates long-term depression (Li et al. 2006). GRF1 can also induce LTP in the CA1 when it is mediated by calcium-permeable AMPA-type glutamate receptors. This occurs via the activation of p38 Map kinase to promote contextual discrimination learning (Jin et al. 2013). GRF1 also induces LTP in the amygdala (Brambilla et al. 1997) and perirhinal cortex (Silingardi et al. 2011), but which receptors activate GRF1 and their role in behavior in these contexts is not clear. GRF1 also contributes to survival of adult-born hippocampal neurons (Darcy et al. 2013). GRF1 expression and function is highly age-dependent, since it plays no detectable role in hippocampal synaptic plasticity until early-adolescence and then continues to contribute throughout adulthood in both males and females (Li et al. 2006).

Because the hippocampus is also known to regulate the HPA axis and glutamate signaling is thought to be involved (Evanson \& Herman 2015), we investigated the role of GRF1 in this process. In this manuscript, we report that GRF1 normally suppresses the HPA axis response to short-term stress and habituation to chronic stress. Remarkably, these defects are observed in early- and mid-adolescent female mice but not in older females or in males, implying that GRF1 is part of previously unknown signaling networks designed to regulate the HPA axis specifically in young females.

\section{Materials and methods}

\section{Animals}

Female and male RasGrf1 homozygous knockout (GRF1 $(-/-)$ ) mice and WT littermate mice, generated as described previously (Giese et al. 2001) and backcrossed onto a C57BL/6j background for more than ten generations, were used in this study. Early-adolescent female and male (pn 28), mid-adolescent female (pn 35), adult female and male (pn 60) mice are used in the studies. All mice were housed in a temperature- and humiditycontroled facility on a reversed $12 \mathrm{~h}$ light: $12 \mathrm{~h}$ darkness cycle with food and water ad libitum. All procedures were

Published by Bioscientifica Ltd. 
carried out in accordance with the Institutional Animal Care and Use Committee guidelines of Tufts University.

\section{Restraint stress}

Stressed mice were placed in plastic restrainer bags for $30 \mathrm{~min} /$ day up to 7 consecutive days. Restrainer bags were cut on the small end to allow the noses to poke through for air and the back of the bag was tied with a twist tie around the tail to prevent escape and movement of mice. In contrast, unstressed control mice remained in their home cage. Immediately after the last stress exposure, mice were either used in behavioral tests or decapitated for blood CORT measurements. Stress procedure was performed between 1200 and $1500 \mathrm{~h}$.

\section{CRH injections}

$150 \mu \mathrm{g} / \mathrm{kg}$ of CRH (Sigma; van Gaalen et al. 2002) or saline (Teknova, Hollister, CA, USA) solutions were injected intraperitoneally between 1000 and $1100 \mathrm{~h}$. After injections, mice were kept in their home cages for $1 \mathrm{~h}$ and trunk blood was collected for CORT measurements.

\section{Behavior tests}

Elevated plus maze test Mice were placed in the center of a plus-shaped maze elevated $40 \mathrm{~cm}$ from the ground, composed of two open and two closed arms, each $35.5 \mathrm{~cm}$ long and $5 \mathrm{~cm}$ wide (Campden Instruments Ltd, Lafayette, IN, USA). General mouse activity was analyzed for $5 \mathrm{~min}$ and the percent time spent in the open arms was recorded using the Motor Monitor Software (Campden Instruments Ltd).

Open field test Mice were placed in a 16-by-16-inch open arena connected to an activity frame (Campden Instruments Ltd) and allowed to move under even illumination for $5 \mathrm{~min}$. General locomotor activity was analyzed and total distance was recorded using the Motor Monitor Software.

\section{Ovariectomy}

Ovariectomies were performed under aseptic conditions under a magnifying lamp to increase visibility. Briefly, mice were anesthetized with ketamine and xylazine, and the ovaries were removed through two small incisions made in the dorsal body wall on each side just under the rib cage. After ligation of the blood supply to the ovary, the ovary was removed with a scalpel. The muscle wall was sutured and the skin was closed with two stainless steel wound clips. For analgesia, Buprivicaine was first splashed into the incision site prior to closing and Buprenorphine was administered subcutaneously following the completion of surgery. Animals were closely observed on heating pads until they were awake and alert and ready to be returned to their racks. All animals were closely monitored for 3 days post surgery.

\section{Stereotactic surgery}

Twenty-four-day-old GRF1(-/-) mice (the youngest we could efficiently target the CA1) were anesthetized with an i.p. injection of ketamine $(100 \mathrm{mg} / \mathrm{kg}) / x y l a z i n e ~(10 \mathrm{mg} / \mathrm{kg})$. Once anesthetized, each mouse was placed in a stereotactic frame (myNeuroLab, St Louis, MO, USA). A surgical incision was made along the midline of the head to expose the skull. Two holes were made in the skull overlying the hippocampus. Coordinates for CA1 injection into the mice: $2.5 \mathrm{~mm}$ posterior to Bregma, $\pm 2.5 \mathrm{~mm}$ lateral from the midline, $1.75 \mathrm{~mm}$ beneath the surface of the skull. Injections were performed with a $10 \mu \mathrm{l}$ Hamilton syringe fitted with a custom-made blunt-ended 30-gauge needle (Hamilton, Reno, NV, USA). Each injection consisted of $1 \mu \mathrm{l}$ of adenovirus expressing various GRF proteins (see Jin et al. $(2013,2014)$ for description of viruses used and how they were generated) infused at a rate of $0.06 \mu \mathrm{l} / \mathrm{min}$. An infusion pump controling the plunger on the Hamilton syringe precisely regulated the rate of injection. The needle was then left in place for $8 \mathrm{~min}$ prior to withdrawal from the brain.

\section{Tissue preparation and sectioning for immunofluorescence}

Seven to ten days after the stereotactic surgeries, mice were deeply anesthetized with ketamine/xylazine and transcardially perfused with $0.1 \mathrm{M}$ phosphate buffer (PB) followed by $4 \%$ paraformaldehyde (PFA 4\%) dissolved in PB $0.1 \mathrm{M}$. Brains were extracted and post-fixed in PFA $4 \%$ for $24 \mathrm{~h}$. Brains were transferred to $30 \%$ sucrose for $48-72 \mathrm{~h}$ before slicing $30 \mu \mathrm{m}$ coronal sections through the extent of the hippocampus using a cryostat. Sections were stored in cryoprotectant at $-20^{\circ} \mathrm{C}$ until use. Each immunohistochemical analysis was conducted from $30 \mathrm{~mm}$ sections spanning the hippocampus.

\section{Immunohistochemistry}

Free-floating sections were rinsed extensively in PBS with $0.25 \%$ Triton X-100 (PBS-T). Sections were blocked for

Published by Bioscientifica Ltd 
$1 \mathrm{~h}$ at room temperature in PBS-T with 5\% normal goat serum. Primary antibody, antiGRF1 (C 18) (Santa Cruz Biotechnology, Santa Cruz, CA, USA), NueN and GFAP, were diluted in the blocking solution (1:200), incubated overnight at $4{ }^{\circ} \mathrm{C}$, and rinsed three times for $15 \mathrm{~min}$ in PBS-T. The sections were then incubated for $1.5 \mathrm{~h}$ at RT with a mixture of Alexa 488 and Cy3-conjugated secondary antibody (1:300; Invitrogen). The stained sections were examined with a Nikon (Tokyo, Japan) fluorescence microscope and images were captured with a CCD spot camera.

\section{CORT measurements}

Stressed and control mice were decapitated and trunk blood was collected individually in $1.5 \mathrm{ml}$ EDTA-coated microcentrifuge tubes (Fisher Scientific, Pittsburg, PA, USA). All blood samples were maintained on ice and centrifuged at $4800 \boldsymbol{g}$ for $15 \mathrm{~min}$ at $4{ }^{\circ} \mathrm{C}$. Plasma was separated and stored at $-80^{\circ} \mathrm{C}$ until analysis. CORT levels in plasma were measured using CORT EIA Kit (Enzo Life Sciences, Farmingdale, NY, USA), according to the manufacturer's instructions. The intra-assay variability ranged from 6.6 to $8.0 \%$, inter-assay variability ranged from 7.8 to $13.1 \%$, and mean assay sensitivity was $26.99 \mathrm{pg} / \mathrm{ml}$. Stressed mice were decapitated immediately after the last stress exposure and none of the stressed or control mice were submitted to any behavioral tests before. All blood samples were collected during lights-on period between 1200 and $1500 \mathrm{~h}$.

\section{Statistical analysis}

All the statistical analyses were performed using Prism 5 Software (GraphPad Prism Software, La Jolla, CA, USA). Student's $t$-tests or ANOVA were used to analyze the differences between stressed and control groups for behavioral analyses and CORT measurements. Post hoc multiple comparisons using Bonferroni's correction were performed. For all comparisons, values of $P<0.05$ were considered significant.

\section{Results}

\section{Generation of a stress response in WT and RasGRF1(-I-) mice}

To begin to test whether RasGRF1 (GRF1) contributes to HPA axis regulation, early-adolescent (pn 28) and adult (pn 60), WT and GRF1(-/-) mice were submitted daily to
30 min restraint stress for 7 days. One indication of a chronic stress response in mice is a decrease in weight gain throughout stress exposure. Weights of all the animals were measured daily for 7 consecutive days and weight gain over 7 days was calculated. Early-adolescent female (Fig. 1A), adult female (Fig. 1B), and early-adolescent male (Fig. 1C) WT and GRF1 $(-/-)$ mice all exhibited a similar decrease in weight gain after 7 days of restraint stress compared to unstressed controls (Fig. 1A, B and C) indicating that they all experienced restraint stress similarly. GRF1 $(-/-)$ males appeared to respond more strongly to this paradigm but as shown below they showed no HPA axis abnormalities.

\section{Early-adolescent female GRF1(-I-) mice have a blocked HPA response to chronic restraint stress}

To test for HPA axis response to this stress paradigm, immediately after the last of 7 daily $30 \mathrm{~min} /$ day restraint stress exposures on a new set of animals, trunk blood CORT levels were measured. We began with earlyadolescent animals because this is the time when GRF proteins begin to contribute to synaptic plasticity (Li et al. 2006). We also began our studies with females. As expected, CORT levels rose approximately three-fold in early-adolescent female WT mice (Fig. 2A, left panel). Strikingly, although baseline CORT levels in earlyadolescent female GRF1(-/-) mice were similar to their WT counterparts, the stress-induced increase in CORT observed in WT mice was completely blocked in GRF1(-/-) mice (Fig. 2A, right panel).

To determine whether this altered HPA axis output in GRF1(-/-) mice was reflected in altered behavior, another set of early-adolescent female WT and GRF1(-/-) mice were tested for anxiety-related behavior in the elevated plus maze (EPM). While one might have assumed that the stressed mice spend less time in the open arms consistent with enhanced anxiety, we found the opposite - they spent more time. However, this is similar to results from previous studies on adolescent mice exposed to restraint stress (Fig. 2B, left panel; Toledo-Rodriguez \& Sandi 2011). Although increased activity in EPM may not be due to increased HPA axis activity, the block in behavior change we find in GRF1 mice (Fig. 2B, right panel) is consistent with a block in HPA axis function.

In the open field test (OFT), total distance traveled increased significantly in stressed adolescent female WT mice compared to the unstressed group, indicative of hyperactive behavior (Marin et al. 2007; Fig. 2C, left panel). This could explain the increased time these mice

Published by Bioscientifica Ltd. 

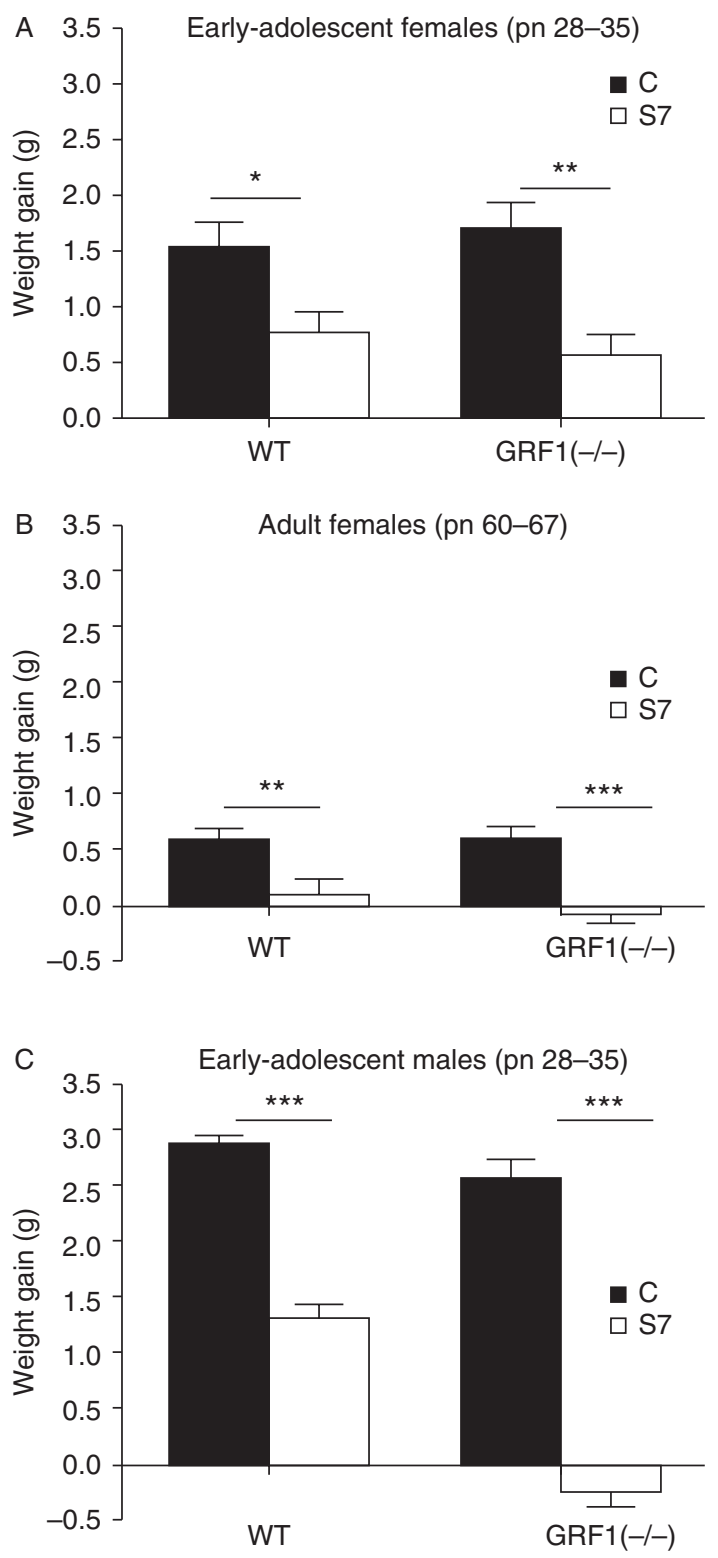

\section{Figure 1}

Similar suppression of weight gain in WT and GRF1(-I-) mice after 7 days of chronic restraint stress. Early-adolescent (pn 28) and adult (pn 60) WT (C) and GRF1(-I-) mice were subjected to restraint stress (30 $\mathrm{min} /$ day) for 7 days (S7) and total weight was then measured (pn 35) (GRF1 mice are $\sim 85 \%$ the size of WT BI6 mice). (A) In early-adolescent females stress had a significant effect on weight gain in both WT and GRF1(-/-) mice (WTC $n=8$, WTS $n=9$, GRF1 $(-l-)$ C $n=8$, and WTS $n=9)\left(F_{(2,30)}=20.21\right.$, $P<0.0001$; Bonferroni post-hoc tests for WT control vs stress, $t=2.528$, $P<0.05$; and GRF1 $(-/-)$ control vs stress, $t=3.829, P<0.01)$. (B) In adult females stress had a significant effect in weight gain in both WT and GRF1 $(-1-)$ mice ( $n=9$ for each group) $\left(F_{(2,32)}=24.46, P<0.0001\right.$; Bonferroni post-hoc tests for WT control vs stress, $t=3.093, P<0.01$; and GRF1(-I-) control vs stress, $t=3.900, P<0.001)$. (C) In early-adolescent males stress had a significant effect in weight gain in WT and GRF1(-/-) mice ( $n=5$ for each group) $\left(F_{(2,16)}=280.05, P<0.0001\right.$; Bonferroni post-hoc tests for WT control vs stress $t=8.445, P<0.001$; and GRF1 $(-/-)$ control vs stress $t=15.22, P<0.001) .{ }^{*}, P<0.05 ; * *, P<0.01 ; * * *, P<0.001$. spent in the open arms of the EPM observed in Fig. 2B, due to their hyperactivity. Similar to the EPM data, this increase in locomotor behavior was also blocked in stressed early-adolescent female GRF1(-/-) mice (Fig. 2C, right panel). These results indicate that, even though these GRF1 $(-/-)$ mice had a similar decrease in weight gain as WT mice (Fig. 1A), they failed to mount an HPA axis response to chronic restraint stress evidenced by elevated CORT levels and enhanced locomotion.

To determine whether this defect was due to altered HPA axis function in the CNS or in the pituitary or adrenal glands, early adolescent WT and GRF1(-/-) female mice were injected intraperitoneally with CRH $(150 \mu \mathrm{g} / \mathrm{kg})$ or saline. One hour later trunk blood was collected for CORT measurements. CRH injection lead to an $\sim 2.5$-fold increase in CORT levels in both WT mice (Fig. 2D, left panel) and GRF1(-/-) mice (Fig. 2D, right panel). Next, GRF1(-/ - ) mice were exposed to 6 days of restraint stress and instead of subjecting to them to the seventh exposure, $\mathrm{CRH}$ was injected. Even though these mice do not display elevated CORT levels when subjected to a seventh restraint stress exposure, they did respond normally when injected with CRH (Fig. 2E). Barring indirect effects from peripheral effects of CRH (Martins et al. 1997), this shows that the defect in these mice is at the level of the hypothalamus or CNS regions that regulate it and not in the pituitary or adrenal gland. This conclusion is consistent with the fact that GRF1 levels are undetectable in the pituitary and adrenal glands (http://www.informatics.jax.org; McCormick et al. 2010).

\section{Role of GRF1 in HPA axis response to chronic restraint stress is age-dependent}

To determine whether the GRF1 contribution to the HPA axis response to stress also continues through adulthood, adult (pn 60) female WT and GRF1(-/-) mice were submitted to 7 days of chronic restraint stress, and then CORT levels and anxiety-related behaviors were measured as described above.

WT adult female mice displayed an increase in CORT levels (Fig. 3A, left panel) that was similar to that observed in early-adolescent female mice (Fig. 2A). Remarkably, unlike their early-adolescent counterparts, stressed adult female GRF1(-/-) mice showed a rise in CORT levels similar to that found in WT mice (Fig. 3A, right panel). Similarly, stressed adult female WT and GRF1(-/-) mice showed similar increases in distance traveled in the OFT, compared to unstressed counterparts (Fig. 3B). Also, stressed adult female WT and GRF1(-/-) mice showed

Published by Bioscientifica Ltd 
Early-adolescent females (pn 20-35)
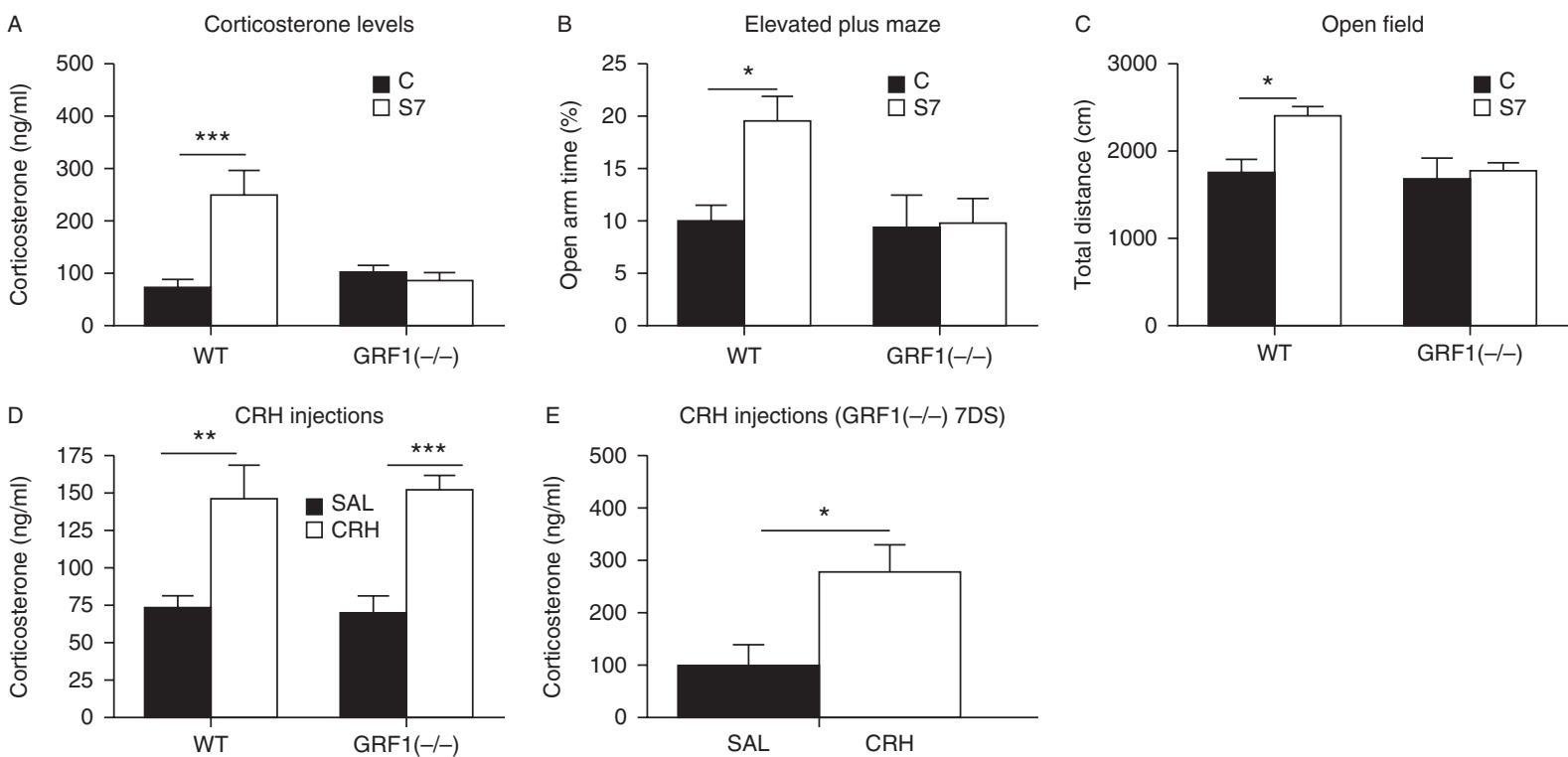

\section{Figure 2}

Failure of early-adolescent female GRF1(-I-) mice to generate a stress response after 7 days of chronic restraint stress. Early-adolescent female WT (C) and GRF1(-I-) mice (pn 28) were subjected to 7 days of chronic restraint stress. Circulating CORT levels were measured or mice were submitted to several behavioral assays for evaluation of anxiety-like phenotype and locomotor behaviors on pn 35. (A) WT mice displayed an increase in CORT levels after stress exposure compared to unstressed counterparts whereas GRF1( $-I-)$ mice displayed blocked CORT response ( $n=6$ in each group) $\left(F_{(2,20)}=7.94, P=0.0106\right.$; Bonferroni post-hoc tests for WT control vs stress, $t=4.381, P<0.001)$; and GRF1( $-I-)$ control vs stress $t=0.395, P>0.05)$. (B) In the elevated plus maze, stressed WT mice spent more time in the open arms compared to unstressed counterparts and stressed GRF1(-/-) mice showed no change in EPM behavior (WTC $n=8$ and WTS7 $n=6$, GRF1C $n=8$ and GRF1S7 $n=8)\left(F_{(2,26)}=3.832, P=0.0611\right.$ Bonferroni post-hoc tests WT control vs stress, $t=2.560, P<0.05$; and

comparable increases in the time they spent in the open arms of the EPM (Fig. 3C).

To better define the developmental period when the HPA axis becomes independent of GRF1, we repeated the experiments described above with mice during midadolescence (pn 35) such that the CORT measurements were performed 7 days later (pn 42). Remarkably, these mid-adolescent female GRF1(-/-) mice displayed a CORT response to 7 days of restraint stress that was comparable to that of WT mice (Fig. 3D). Thus, the HPA axis in female mice transitions from GRF1-dependent to GRF1-independent during a $\sim 1$-week period when mice transition from early- to mid-adolescence.

The time period between early-adolescence and mid-adolescence when HPA axis becomes independent of GRF1 is when $\mathrm{C} 57 \mathrm{Bl} / 6 \mathbf{j}$ mice enter puberty. To determine if the rise in estrogen after puberty is the
GRF1 $(-/-)$ control vs stress $(t=0.116, P>0.05)$. (C) In the open field test, stressed WT mice travel more distance in the open field and stressed GRF1(-I-) mice show no change in the total distance traveled (WTC $n=8$ and WTS7 $n=6$; GRF1C $n=8$ and GRF1S7 $n=8)\left(F_{(2,26)}=5.138, P=0.0320\right.$; Bonferroni post-hoc tests for WT control vs stress, $t=2.663, P<0.05$; and GRF1 ( $-I-$ ) control vs stress, $t=0.4602, P>0.05)$. (D) $150 \mu \mathrm{g} / \mathrm{kg}$ of $\mathrm{CRH}$ was injected intraperitoneally to WT and GRF1(-l-) mice. Both of the groups showed a significant increase in CORT secretion upon CRH injection (WT-SAL $n=5$, WT-CRH $n=5$, GRF1-SAL $n=5$, and GRF1-CRH $n=6)\left(F_{(2,17)}=65.85\right.$, $P<0.0001$; Bonferroni post-hoc tests for WT SAL vs CRH, $t=3.773, P<0.01$; and GRF1(-l-) SAL vs CRH, $t=4.447, P<0.001$ ). (E) Early-adolescent GRF1(-I-) females were submitted to 6 days of restraint stress and on day 7 one dose $(150 \mu \mathrm{g} / \mathrm{kg})$ of CRH was injected to determine CORT levels.

Secreted CORT was increased significantly in GRF1 $(-/-)$ mice (SAL $n=3$ and $\mathrm{CRH} n=5)\left(t_{6}=2.603, P=0.0405\right) . *{ }^{*} P<0.05 ; * *, P<0.01 ; * * *, P<0.001$.

cause of the independence from GRF1, early-adolescent GRF1(-/-) females were ovariectomized (OVX) and submitted to 7 days of restraint stress when they reach 2 months of age. Figure 3E shows that OVX-GRF1 $(-/-)$ mice display similar basal CORT levels as non-OVX counterparts and a similar increase in CORT response to 7 days of restraint stress. Thus, the change from GRF1 dependence before puberty to GRF1 independence in adulthood is due to some developmental process not dependent upon elevated levels of estrogen that begins during puberty.

\section{Role of GRF1 in HPA axis response to chronic restraint stress is sex-dependent}

To determine whether GRF1 also contributes to HPA axis response to chronic restraint stress in males,

Published by Bioscientifica Ltd. 

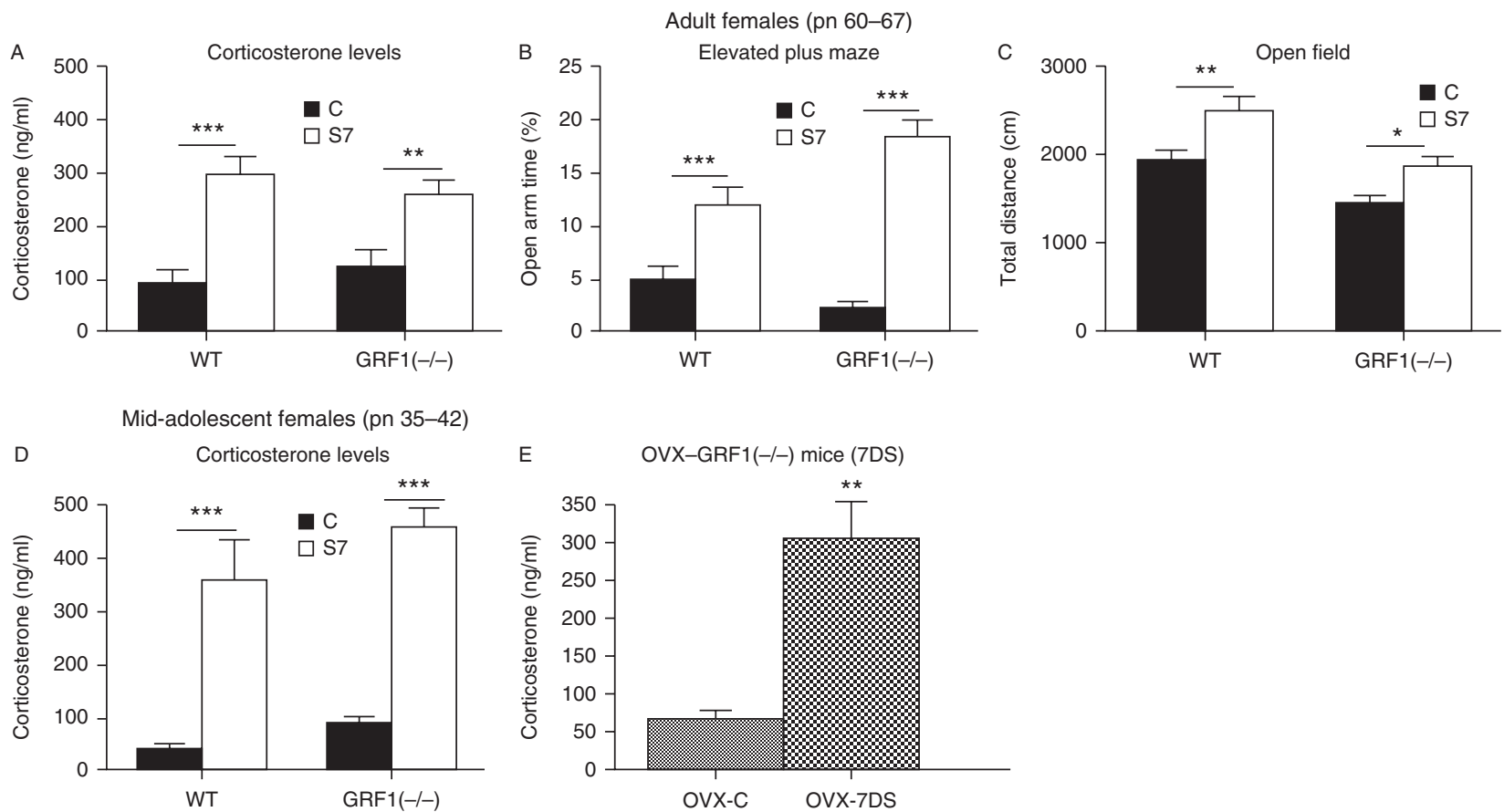

Figure 3

Normal chronic restraint stress response in middle-adolescent and adult female GRF1(-I-) mice. Mice were submitted to 7 days of chronic restraint stress (S7). CORT levels were measured or anxiety-related behaviors were tested. (A) Stressed WT (C) and GRF1 (-I-) adult (pn 60) females displayed a similar increase in their circulating CORT levels compared to unstressed counterparts ( $n=5$ for each group) $\left(F_{(2,16)}=35.76, P<0.0001\right.$; Bonferroni post-hoc tests for WT control vs stress, $t=5.073, P<0.001$; and GRF1( $-/-$ ) control vs stress, $t=3.384, P<0.01)$. (B) In OFT, stressed WT and stressed GRF1 ( $-I-)$ adult females showed a significant increase in the total distance traveled compared to their unstressed counterparts ( $n=6$ for each group) $\left(F_{(2,20)}=19.92, P<0.0001\right.$; Bonferroni post-hoc tests for WT control vs stress, $t=3.617, P<0.01$; and GRF1 $(-1-)$ control vs stress, $t=2.696, P<0.05)$. (C) In EPM, adult female WT mice spent significantly more time in the open arms after stress exposure. A similar increase in the open arm time was observed for stressed GRF1 $(-/-)$ females compared to their unstressed counterparts ( $n=8$ for each group) $\left(F_{(2,28)}=88.57, P<0.0001\right.$; Bonferroni post-hoc tests for WT control vs stress, $t=4.074, P<0.001$; and GRF1( $-I-$ ) control vs stress, $t=9.235, P<0.001$ ). (D) Stressed mid-adolescent (pn 35) WT (control $n=5$ and stress $n=3$ ) and GRF1 $(-l-$ ) (control $n=5$ and stress $n=5$ ) females displayed a significant increase in circulating CORT levels compared to their unstressed counterparts $\left(F_{(2,14)}=117.8, P<0.0001\right.$; Bonferroni post-hoc tests for WT control vs stress, $t=6.709, P<0.001$; and GRF1 $(-I-)$ control vs stress, $t=8.831, P<0.001)$. (E) Ovariectomy of GRF1 $(-I-)$ females did not alter the basal CORT levels and CORT response after chronic stress, $n=3$ for each group $\left(t_{4}=4.939, P=0.0078\right)$. ${ }^{*}, P<0.05 ; * *, P<0.01 ; * * *, P<0.001$. early-adolescent and adult male WT and GRF1(-/-) mice were submitted to 7 days of $30 \mathrm{~min} /$ day restraint stress. Like their female counterparts, WT male mice displayed a large increase in CORT levels after the seventh exposure to stress (Fig. 4A, left panel). In contrast to their female counterparts, early-adolescent male GRF1 $(-/-)$ mice displayed a CORT response similar to WT mice (Fig. 4A, right panel). Adult male GRF1(-/-) mice also showed a normal CORT response (Fig. 4B). Thus, not only is the role of GRF1 in regulating the HPA axis age-dependent, it is also sexdependent, such that it represents a distinct regulator only in early-adolescent females. Similar behavior studies were performed on early-adolescent and adult male mice; however, no differences in behavior were detected even in stressed WT groups (data not shown).

\section{Enhanced short-term HPA response to restraint stress specifically in early- to mid-adolescent female GRF1(-I-) mice}

To determine whether the results observed after chronic restraint stress are due to the fact that early-adolescent female GRF1 (-/-) mice have a completely defective HPA axis, these mice were tested for an acute stress response by measuring CORT levels immediately after a single $30 \mathrm{~min}$ exposure to restraint stress. Figure 5A, S1 shows this is not the case. While early-adolescent female WT mice displayed a approximately twofold increase in CORT levels after acute restraint stress, their GRF1(-/-) counterparts actually showed an enhanced CORT response reaching a approximately fourfold rise. Thus, not only do GRF1 $(-/-)$ mice have a functional acute response to restraint stress, it is magnified.

Published by Bioscientifica Ltd. 

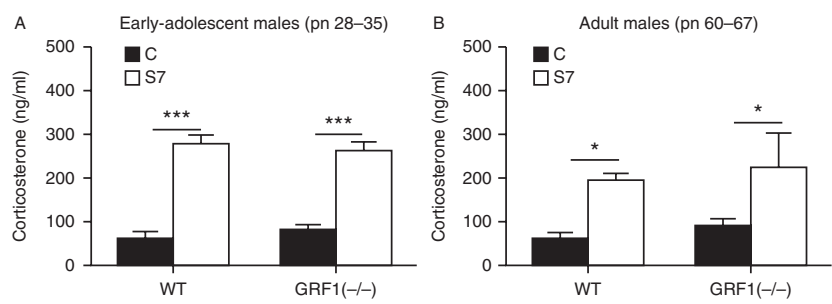

Figure 4

Normal chronic restraint stress response in early-adolescent male and adult male GRF1 $(-I-)$ mice. Mice were submitted to 7 days of chronic restraint stress (S7) and CORT levels were then measured. (A) Early-adolescent male WT mice showed significantly increased CORT levels upon stress exposure compared to unstressed counterparts (WT control $n=5$ and stress $n=2$ ). A similar increase was observed with stressed early-adolescent male GRF1 $(-/-)$ males (GRF1 $(-/-)$ control $n=5$ and stress $n=5)\left(F_{(2,13)}=120.6\right.$, $P<0.0001$; Bonferroni post-hoc tests for WT control vs stress, $t=7.057$, $P<0.001$; and GRF1 ( $-I-$ ) control vs stress, $t=6.732, P<0.001)$. (B) Adult male WT (control $n=3$ and stress $n=4$ ) and GRF1 $(-l-$ ) (control $n=4$ and stress $n=3$ ) males displayed a similar increase in CORT levels after 7 days of stress exposure compared to unstressed counterparts $\left(F_{(2,10)}=15.95, P=0.0025\right.$; Bonferroni post-hoc tests for WT control vs stress, $t=2.831, P<0.05$; and GRF1 ( $-I-$ ) control vs stress, $t=2.816, P<0.05)$. ${ }^{*}, P<0.05 ; * * *, P<0.001$.

To better understand the transition in the role of GRF1 from normally suppressing the HPA response after one stress exposure to promoting it after seven stress exposures, the consequences of exposure numbers in between were determined. Figure 5A shows that WT mice maintained elevated CORT levels (approximately twofold) after 3 (S3), 5 (S5) and 7 (S7) days of daily exposures to $30 \mathrm{~min} /$ day restraint stress (the level at day 7 appears slightly higher but it is not statistically different). This finding is consistent with a previous study showing that mice do not habituate to $30 \mathrm{~min} /$ day of restraint stress for at least 10 days (Rademacher et al. 2008). In contrast, early-adolescent female GRF1(-/-) mice displayed an even more-magnified CORT response (approximately six-fold) compared to non-stressed mice after 3 days of restraint stress (S3). However, the mice became less responsive (approximately fivefold) after 5 exposures (S5) and no longer responded after 7 exposures (S7) as described in Fig. 1 above. These results show that earlyadolescent GRF1(-/-) females can, in fact, respond to restraint stress, but that they habituate to it more rapidly than WT mice.

This set of experiments was then repeated on adult female, early-adolescent male, and adult male mice to reveal whether defective responses of GRF1(-/-) mice to fewer exposures to restraint stress are also female and early-adolescence specific. Figure $5 \mathrm{~B}, \mathrm{C}$ and $\mathrm{D}$ shows that this is the case because the responses of both of these sets of GRF1(-/-) mice at the maximum and minimum time points were similar to WT mice. However, we can't eliminate the possibility that these mice show enhanced CORT response for a short-time period between these points. Interestingly, when mid-adolescent mice (pn 35)
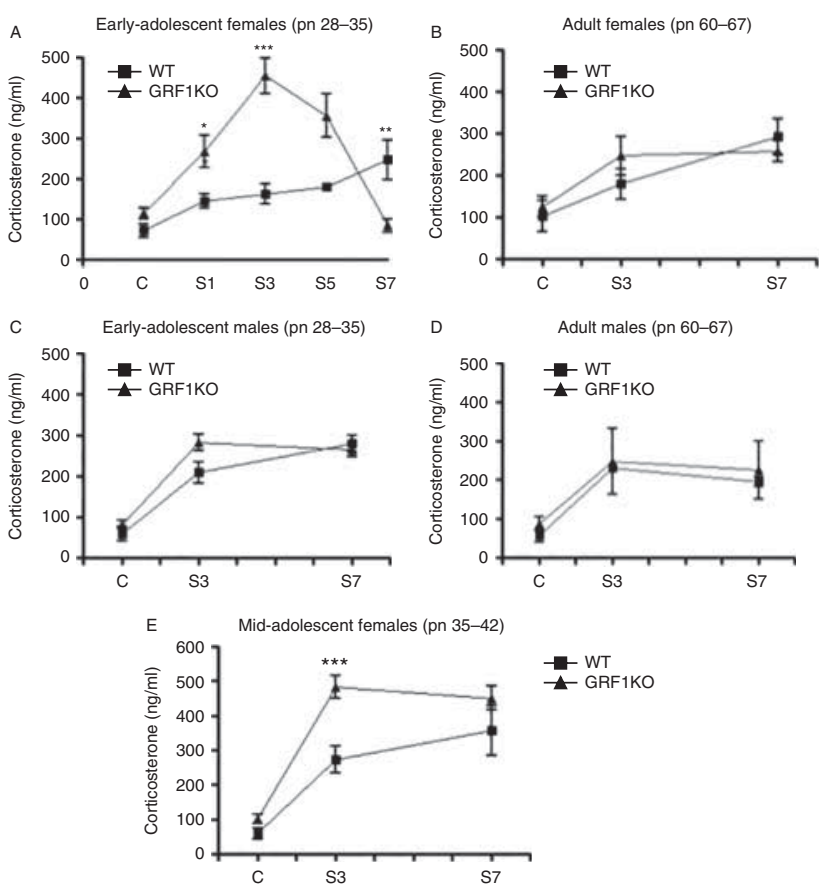

Figure 5

Enhanced short-term restraint stress response specifically in early- to midadolescent female GRF1(-I-) mice. (A) Early-adolescent female WT (C); and GRF1 (-/-) mice were submitted to $1,3,5$, and 7 days of restraint stress (S1, S3, S5, and S7) (30-min/day) and then CORT levels were determined. GRF1(-I-) mice displayed enhanced short-term stress response compared to WT mice until day 5 followed by a blocked response at day 7 (WT C $n=9$, S1 $n=8$, S3 $n=9$, S5 $n=3$, S7 $n=6$ and GRF1 $(-/-)$ C $n=9$, S1 $n=7$, S3 $n=10$, S5 $n=3$, S7 $n=6)\left(F_{(2,60)}=17.80, P<0.001\right.$; Bonferroni post-hoc tests for WT vs GRF1( $-I-)$, C $t=1.05, P>0.05$; S1 $t=2.789, P<0.05$; S3 $t=7.445$, $P<0.001$, S5 $t=2.523, P>0.05$, S7 $t=3.319, P<0.01)$. (B) Adult GRF1( $-/-$ ) females displayed normal short-term restraint stress response compared to WT counterparts (WT C $n=6, \mathrm{~S} 3=3, \mathrm{~S} 7=4$ and GRF1(-I-) C $n=6, \mathrm{~S} 3 n=3$, S7 $n=5)\left(F_{(2,21)}=0.370, P=0.5475\right.$; Bonferroni post-hoc tests WT vs GRF1 ( $-I-), C t=0.520, P>0.05$; S3 $t=1.117, P>0.05$; S7 $t=0.710, P>0.05)$. (C) Early-adolescent GRF1(-I-) males displayed normal short-term restraint stress response compared to their WT counterparts (WT C $n=5$, S3 $n=3$, S7 $n=2$ and GRF1( $-l-)$ C $n=5$, S3 $n=4$, S7 $n=5)\left(F_{(2,18)}=2.733\right.$, $P=0.1156$; Bonferroni post-hoc tests for WT vs GRF1(-I-) C, $t=0.914$, $P>0.05$; S3 $t=2.574, P>0.05$; S7 $t=0.477, P>0.05)$. (D) Adult GRF1(-I-) males also displayed normal short-term restraint stress response on day 3 of restraint stress exposure compared to their WT counterparts (WT C $n=3$, S3 $n=2$, S7 $n=4$ and GRF1 $(-I-)$ C $n=4$, S3 $n=3$, S7 $n=3)\left(F_{(2,13)}=0.4732\right.$, $P=0.5036$; Bonferroni post-hoc tests for WT vs GRF1(-I-) C, $t=0.4997$, $P>0.05$; S3, $t=0.2393, P>0.05$; $57 t=0.4880, P>0.05$ ). (E) Mid-adolescent (pn 35) WT and GRF1(-/-) females were exposed to 3 and 7 days of restraint stress. Both displayed normal stress behavior on day 7 (pn 42). However, on day 3, in mid-adolescence, GRF1(-I-) mice showed enhanced stress response (WT C $n=7$, S3 $n=5$, S7 $n=3$ and GRF1 $(-/-)$ C $n=7$, S3 $n=5$, S7 $n=5)\left(F_{(2,26)}=20.32, P=0.001\right.$; Bonferroni post-hoc tests for WT vs GRF1( $-I-)$ C, $t=1.11, P>0.05$; S3 $t=4.766, P<0.001$; S7 $t=1.874$, $P>0.05)$. * $P<0.05 ; * *, P<0.01 ; * * *, P<0.001$. 
were tested after 3 and 7 days of restraint stress, no significant difference between WT and GRF1(-/-) mice was observed on day 7 (Fig. 5E). They still showed an enhanced response at 3-days of stress in GRF1 (-/-) mice, which might indicate that the transition from GRF1dependent to GRF1 independent HPA axis in females occurs between 38 and 42 days of age. These findings also show that an exaggerated CORT response after 3 days of stress does not necessarily lead to suppressed CORT response after 7 days (see Fig. 5).

\section{GRF1 function in the hippocampus is responsible for its regulation of the HPA axis response to short-term restraint stress}

Because GRF1(-/-) mice display enhanced HPA axis response to short-term stress, we hypothesized that it normally contributes to a negative feedback mechanism that is defective in these mice. Since the hippocampus is one of the key regulators of this mechanism and our lab showed previously that GRF1 regulates synaptic plasticity there, we focused first on the hippocampus. We previously published (Jin et al. 2014) that we can reconstitute all of the known synaptic plasticity functions of GRF1 in GRF1(-/-) mice by stereotactic injection of adenoviruses expressing GRF1 into the CA1 region of the hippocampus. We also described a GRF1 mutant, $\left(\mathrm{PCQ}_{2}\right) \mathrm{GRF} 1$, that expresses at the same level as WT GRF1 but has none of the synaptic plasticity functions of GRF1 (see Fig. 1 and Table 1 from Jin et al. (2014)). Thus, we infected the ventral CA1 of GRF1( $-/-)$ mice with virus expressing either WT GRF1 (GRF1-AV) or inactive GRF1 ( $\left.\mathrm{PCQ}_{2}\right) \mathrm{GRF} 1$ and waited for 7 days, a period we showed previously allowed both forms of GRF1 to restore GRF1 expression to levels found in WT mice (Jin et al. 2014). After expression, co-staining of GRF with either the neuronal marker, NeuN, or glia marker, GFAP, showed that GRF proteins were found overwhelmingly in neurons, not glia, consistent with the neuronspecific synapsin promoter used in these experiments and the known localization of endogenous GRF proteins (Zippel et al. 1997; data not shown).

Then, the injected mice were exposed to 3 days of restraint stress and CORT levels were measured. Figure $6 \mathrm{~A}$, $B$ and $C$ shows that the GRF1 proteins expressed as expected. Importantly, Fig. 6D shows that CORT levels in $\operatorname{GRF} 1(-/-)$ mice reconstituted with a catalytically dead GRF1 mutant (second bar) were super-elevated to a level comparable to those found in uninjected stressed GRF1(-/-) mice (first bar, also see Fig. 5A, S3). However, stressed GRF1(-/-) mice reconstituted with functional
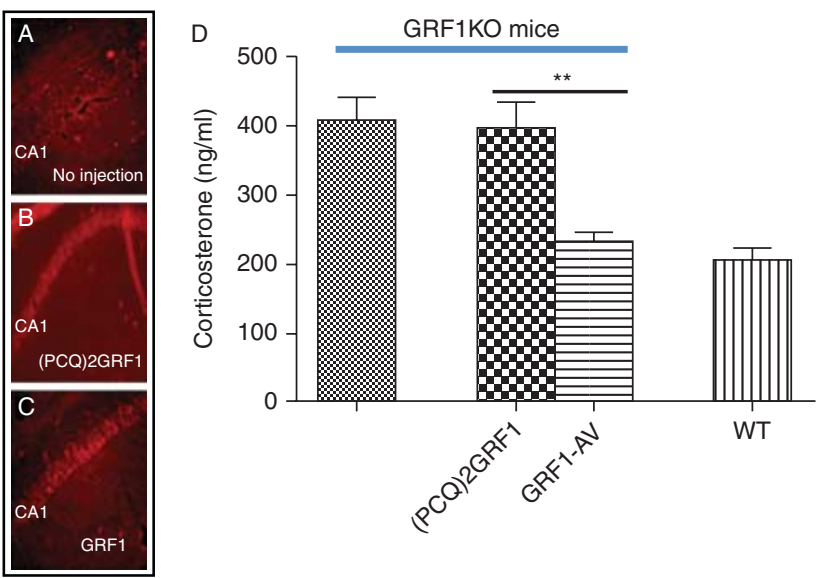

Figure 6

Restoration of GRF1 expression in the ventral CA1 of GRF1 $(-/-)$ mice corrects the defective HPA response to short-term stress. GRF1 staining of the CA1 hippocampus from (A) uninjected pn 24 female GRF1(-/-) mice, or comparable mice stereotactically injected into the ventral CA1 with (B) (PCQ) 2 GRF1-AV, or (C) GRF1-AV. Seven days after injection (shown previously to allow expression of exogenous GRF1 and (PCQ) ${ }_{2} \mathrm{GRF} 1$ to levels similar to endogenous levels in WT mice (Jin et al. 2014)), mice were submitted to 3 days of restraint stress and tissues stained for GRF1 levels. (D) Serum CORT levels were measured and their levels in GRF1, but not (PCQ) ${ }_{2} \mathrm{GRF} 1$, reconstituted GRF1(-I-) mice were similar to levels found in stressed WT mice, $n=5$ for each group $\left(F_{(1,16)}=17.21, P<0.0001\right.$; Bonferroni post-hoc tests for (PCQ) ${ }_{2}$ GRF1-AV vs GRF1-AV, $t=4.523, P<0.01$; GRF1 vs (PCQ) ${ }_{2}$ GRF1-AV, $t=0.2937, P>0.05$; and GRF1-AV vs WT, $t=0.7564$, $P>0.05) . * *, P<0.01$. A full colour version of this figure is available via http://dx.doi.org/10.1530/JOE-15-0304

GRF1 (third bar) displayed a CORT response that was back to that found in stressed WT mice (fourth bar). These findings show that the CA1 hippocampus is the main site where GRF1 regulates the HPA axis response to short-term stress.

\section{Discussion}

The Ras and Rac GTPase-activator, GRF1, has been studied extensively in adult mice in the context of its role in controlling glutamate receptor-induced synaptic plasticity in the hippocampus, amygdala, and perirhinal cortex, all of which contribute to learning and memory and dopamine signaling in the striatum that influences L-DOPA induced dyskinesia (Feig 2011). Here, we demonstrate a new function for GRF1 as a regulator of the HPA axis response to stress specifically in early- to midadolescent females. In particular, early-adolescent female GRF1(-/-) mice, but not their early-adolescent male, adult male, mid-adolescent, or adult female counterparts, display defective HPA response after chronic restraint stress. After up to three episodes of $30 \mathrm{~min}$ of daily restraint stress, these GRF1(-/-) mice show a magnified

Published by Bioscientifica Ltd. 
CORT response. This effect is seen only through midadolescence. However, by the fifth episode the response begins to recede and by the seventh these mice no longer respond to restraint stress with elevated CORT levels or enhanced locomotion. In contrast, adult mice do not even begin to habituate to restraint stress after ten daily episodes (Rademacher et al. 2008), and adolescents are even more resistant than adults (Goldman et al. 1973, Romeo \& McEwen 2006).

These findings imply that GRF1 normally functions to suppress the process of habituation to repeated restraint stress. Habituation is normally not permanent, such that after a few weeks an HPA response returns (Grissom \& Bhatnagar 2009). Adolescent female GRF1(-/-) mice regain responsiveness to restraint stress as early as 7 days after cessation of stress exposure (data not shown). However, this also coincides with the developmental transition to mid-adolescence when female GRF1(-/-) mice show a normal habituation, so it is not possible to assign this reemergence of responsiveness to its reversal. The fact that the short-term HPA response is exaggerated in GRF1 $(-/-)$ mice raises the possibility that this drives an accelerated habituation to chronic stress. This is unlikely because it has been demonstrated that the stronger the stress response, the slower the habituation (Grissom \& Bhatnagar 2009). Moreover, the short-term stress response in mid-adolescent females was exaggerated but the mice did not display accelerated habituation to chronic stress. Interestingly, the rate of habituation can be increased in other contexts. For example, this occurs after the second of a repeated series of stresses, by elevating the frequency of stress exposures, or by reducing stress magnitude (Grissom \& Bhatnagar 2009). However, the biochemical mechanisms involved in how the rate of habituation is regulated have not been revealed. The results from the present study implicate GRF1 in this process, but only in young females.

The age-dependence of the role of GRF1 in HPA axis regulation is striking, as it disappears after $\sim 40$ days of age, despite the fact that GRF1 continues to play a role in learning and memory throughout adulthood. The time period, between 28 days when the HPA axis is dependent upon GRF1 and 40 days when it is not, is the transition from early- through mid-adolescence when $\mathrm{C} 57 \mathrm{Bl} / 6 \mathrm{j}$ mice enter puberty. This raises the possibility that the rise in estrogen that occurs in mid-adolescence plays an active role making the HPA axis independent of GRF1. This does not seem to be the case because ovariectomy of GRF1(-/-) mice at 28 days of age did not restore the rapid habituation to 7 days restraint stress in 2-month-old mice that occurs in early-adolescent animals. Rather, hormone-independent development of specific components of the HPA axis at the onset of puberty is likely involved. Because we could not perform restraint stress on animals before weaning it remains possible that GRF1 is also involved in regulating the HPA axis in more juvenile and neonatal animals, a possibility that will require a different stress paradigm to test.

Many studies have shown that adolescent animals respond differently to stress than adults (Romeo 2010, Romeo et al. 2013, Panagiotakopoulos \& Neigh 2014), but distinctions between early-, mid-, and late-adolescence have not been studied in detail. Early-adolescence in mice (pn 21-34) is thought to be similar to human ages of 10-14 (Burke \& Miczek 2014). It is a period of rapid brain development and high vulnerability to excessive stress (Andersen \& Teicher 2008, Romeo 2010). Animal and human studies have observed differences between these two developmental stages in response to specific environmental perturbations. Risk of depression upon early experience was shown to be maximal around earlyadolescence and decreases as humans age through midand late-adolescence (Andersen \& Teicher 2008). In addition, many studies have focused on sex differences in HPA axis response to stress and to responses to treatments for psychiatric disorders. Studies have shown sex differences in the development of depressive symptoms during early-, mid-, and late-adolescence among boys and girls (Dekker et al. 2007). And women are more likely than men to suffer from depression (Marcus et al. 2005, Bangasser \& Valentino 2014) and respond better to a certain class of antidepressants than others (Kornstein et al. 2000). Also, studies have consistently found PTSD to be more common in women than in men, and some studies have found that women with PTSD respond more robustly to SSRI antidepressants than men (Brady \& Back 2002). However, few studies have focused specifically on adolescent females, and fewer on early-adolescent females. One example showed that upon chronic stress, adolescent female mice display blunted stress response compared to their male counterparts due in part to differential transport of GRs to the nucleus (Bourke et al. 2013).

How and why GRF1 controls signaling pathways in cells that control the HPA axis specifically in earlyadolescent females remains to be determined. GRF1 may regulate the HPA axis through its known ability to couple NMDA and calcium-permeable glutamate receptors to both the Erk, p38 Map kinases, since various stress paradigms have been observed to alter the activities of these kinases (Cole et al. 2000, Revest et al. 2005, Ferland et al. 2014).

Published by Bioscientifica Ltd 
However, to date these findings are just correlative and they were detected in adult males. Thus, GRF1 may contribute specificity to HPA axis regulation in earlyadolescent females by coupling these kinases or other downstream effectors to distinct upstream signals that allow the HPA axis of early-adolescent females to respond to unique environmental stimuli.

GRF1(-/-) mice display a complex phenotype, with an enhanced HPA axis response after short-term restraint stress, but blocked HPA axis response after chronic restraint stress. This may reflect different functions of GRF1 in multiple brain regions. In fact, we show that the magnified CORT response observed in these animals after 3 days of restraint stress is due to the loss of GRF1 in the CA1 hippocampus, a region known to play a negative role in regulating the HPA axis (Jankord \& Herman 2008). Thus, GRF1 and presumably GRF1 regulated signaling cascades represent newly identified mediators of the inhibitory role the hippocampus plays on HPA axis response to stress that is functional specifically in adolescent females.

We also showed that block in CORT response to chronic restraint stress in GRF1 $(-/-)$ mice is not due to defects in the pituitary or adrenal gland function but to components of the HPA axis in the CNS. It could be due to GRF1 loss in dentate gyrus of the hippocampus, the PVN, mPFC, and/or the amygdala because all have been reported to play a positive role in promoting habituation (Herman 2013). However, all of the studies identifying the roles of specific brain regions in regulating the HPA axis used adult males, so that novel regions may be involved in early-adolescent females. To test these ideas, the consequence of restoring GRF1 or knocking it out in specific brain regions needs to be investigated, a process that is now underway.

Overall, these findings reveal that the HPA axis in earlyand mid-adolescent females is regulated by a distinct mechanism. In addition, these findings highlight the idea that young females may respond to stressful environments as well as to treatments for stress-induced psychiatric disorders involving the HPA axis in unique ways.

\section{Declaration of interest}

The authors declare that there is no conflict of interest that could be perceived as prejudicing the impartiality of the research reported.

\section{Funding}

Grant sponsor: National Institutes of Health; grant number: RO1 MH083324 (to L A F) and grant sponsor: Tufts Center for Neuroscience Research; grant number: P30 NS047243.

\section{References}

Andersen SL \& Teicher MH 2008 Stress, sensitive periods and maturational events in adolescent depression. Trends in Neurosciences 31 183-191. (doi:10.1016/j.tins.2008.01.004)

Bangasser DA \& Valentino RJ 2014 Sex differences in stress-related psychiatric disorders: neurobiological perspectives. Frontiers in Neuroendocrinology 35 303-319. (doi:10.1016/j.yfrne.2014.03.008)

Barha CK, Brummelte S, Lieblich SE \& Galea LA 2011 Chronic restraint stress in adolescence differentially influences hypothalamic-pituitaryadrenal axis function and adult hippocampal neurogenesis in male and female rats. Hippocampus 21 1216-1227. (doi:10.1002/hipo.20829)

Bhatnagar S, Huber R, Nowak N \& Trotter P 2002 Lesions of the posterior paraventricular thalamus block habituation of hypothalamic-pituitary-adrenal responses to repeated restraint. Journal of Neuroendocrinology 14 403-410. (doi:10.1046/j.0007-1331.2002.00792.x)

Bourke CH, Raees MQ, Malviya S, Bradburn CA, Binder EB \& Neigh GN 2013 Glucocorticoid sensitizers Bag1 and Ppid are regulated by adolescent stress in a sex-dependent manner. Psychoneuroendocrinology 38 84-93. (doi:10.1016/j.psyneuen.2012.05.001)

Brady KT \& Back SE 2002 Gender and the psychopharmacological treatment of PTSD. In Gender and PTSD, pp 335-348. Eds R Kimerling, P Ouimette \& J Wolfe. New York, NY, USA: Guilford Publications.

Brambilla R, Gnesutta N, Minichiello L, White G, Roylance AJ, Herron CE, Ramsey M, Wolfer DP, Cestari V, Rossi-Arnaud C et al. 1997 A role for the Ras signalling pathway in synaptic transmission and long-term memory. Nature 390 281-286. (doi:10.1038/36849)

Burke AR \& Miczek KA 2014 Stress in adolescence and drugs of abuse in rodent models: role of dopamine, CRF, and HPA axis. Psychopharmacology 231 1557-1580. (doi:10.1007/s00213-013-3369-1)

Cole MA, Kalman BA, Pace TW, Topczewski F, Lowrey MJ \& Spencer RL 2000 Selective blockade of the mineralocorticoid receptor impairs hypothalamic-pituitary-adrenal axis expression of habituation. Journal of Neuroendocrinology 12 1034-1042. (doi:10.1046/j.1365-2826. 2000.00555.x)

Darcy MJ, Trouche S, Jin SX \& Feig LA 2013 Age-dependent role for ras-GRF1 in the late stages of adult neurogenesis in the dentate gyrus. Hippocampus 24 315-325. (doi:10.1002/hipo.22225)

Dekker MC, Ferdinand RF, van Lang ND, Bongers IL, van der Ende J \& Verhulst FC 2007 Developmental trajectories of depressive symptoms from early childhood to late adolescence: gender differences and adult outcome. Journal of Child Psychology and Psychiatry, and Allied Disciplines 48 657-666. (doi:10.1111/j.1469-7610.2007.01742.x)

Evanson NK \& Herman JP 2015 Metabotropic glutamate receptor-mediated signaling dampens the HPA axis response to restraint stress. Physiology \& Behavior [in press]. (doi:10.1016/j.physbeh.2015.02.027)

Feig LA 2011 Regulation of neuronal function by Ras-GRF exchange factors. Genes \& Cancer 2 306-319. (doi:10.1177/1947601911408077)

Ferland CL, Harris EP, Lam M \& Schrader LA 2014 Facilitation of the HPA axis to a novel acute stress following chronic stress exposure modulates histone acetylation and the ERK/MAPK pathway in the dentate gyrus of male rats. Endocrinology 155 2942-2952. (doi:10.1210/en.2013-1918)

van Gaalen MM, Reul JH, Gesing A, Stenzel-Poore MP, Holsboer F \& Steckler T 2002 Mice overexpressing CRH show reduced responsiveness in plasma corticosterone after a5-HT1A receptor challenge. Genes, Brain, and Behavior 1 174-177. (doi:10.1034/j.1601-183X. 2002.10305.x)

Giese KP, Friedman E, Telliez JB, Fedorov NB, Wines M, Feig LA \& Silva AJ 2001 Hippocampus-dependent learning and memory is impaired in mice lacking the Ras-guanine-nucleotide releasing factor 1 (Ras-GRF1). Neuropharmacology 41 791-800. (doi:10.1016/S0028-3908(01)00096-X)

Goel N, Workman JL, Lee TT, Innala L \& Viau V 2014 Sex differences in the HPA axis. Comprehensive Physiology 4 1121-1155. (doi:10.1002/cphy. c130054)

Published by Bioscientifica Ltd 
Goldman L, Winget C, Hollingshead GW \& Levine S 1973 Postweaning development of negative feedback in the pituitary-adrenal system of the rat. Neuroendocrinology 12 199-211. (doi:10.1159/000122169)

Grissom N \& Bhatnagar S 2009 Habituation to repeated stress: get used to it. Neurobiology of Learning and Memory 92 215-224. (doi:10.1016/j.nlm. 2008.07.001)

Grissom NM \& Bhatnagar S 2011 The basolateral amygdala regulates adaptation to stress via $\beta$-adrenergic receptor-mediated reductions in phosphorylated extracellular signal-regulated kinase. Neuroscience 178 108-122. (doi:10.1016/j.neuroscience.2010.12.049)

Hall BS \& Romeo RD 2014 The influence of poststress social factors on hormonal reactivity in prepubertal male rats. Developmental Psychobiology 56 1061-1069. (doi:10.1002/dev.21190)

Handwerger K 2009 Differential patterns of HPA activity and reactivity in adult posttraumatic stress disorder and major depressive disorder. Harvard Review of Psychiatry 17 184-205. (doi:10.1080/ 10673220902996775)

Herman JP 2013 Neural control of chronic stress adaptation. Frontiers in Behavioral Neuroscience 7 61. (doi:10.3389/fnbeh.2013.00061)

Jankord R \& Herman JP 2008 Limbic regulation of hypothalamo-pituitaryadrenocortical function during acute and chronic stress. Annals of the New York Academy of Sciences 1148 64-73. (doi:10.1196/annals. 1410.012)

Jin SX, Arai J, Tian X, Kumar-Singh R \& Feig LA 2013 Acquisition of contextual discrimination involves the appearance of a Ras-GRF1/p38 Map kinase-mediated signaling pathway that promotes LTP. Journal of Biological Chemistry 288 21703-21713. (doi:10.1074/jbc.M113.471904)

Jin SX, Bartolome C, Arai JA, Hoffman L, Uzturk BG, Kumar-Singh R, Waxham MN \& Feig LA 2014 Domain contributions to signaling specificity differences between Ras-guanine nucleotide releasing factor (Ras-GRF) 1 and Ras-GRF2. Journal of Biological Chemistry 289 16551-16564. (doi:10.1074/jbc.M114.557959)

Kornstein SG, Schatzberg AF, Thase ME, Yonkers KA, McCullough JP, Keitner GI, Gelenberg AJ, Davis SM, Harrison WM \& Keller MB 2000 Gender differences in treatment response to sertraline versus imipramine in chronic depression. American Journal of Psychiatry $\mathbf{1 5 7}$ 1445-1452. (doi:10.1176/appi.ajp.157.9.1445)

Li S, Tian X, Hartley DM \& Feig LA 2006 Distinct roles for Ras-guanine nucleotide-releasing factor 1 (Ras-GRF1) and Ras-GRF2 in the induction of long-term potentiation and long-term depression. Journal of Neuroscience 26 1721-1729. (doi:10.1523/JNEUROSCI.3990-05.2006)

Marcus SM, Young EA, Kerber KB, Kornstein S, Farabaugh AH, Mitchell J, Wisniewski SR, Balasubramani GK, Trivedi MH \& Rush AJ 2005 Gender differences in depression: findings from the STAR ${ }^{*} \mathrm{D}$ study. Journal of Affective Disorders 87 141-150. (doi:10.1016/j.jad.2004.09.008)

Marin MT, Cruz FC \& Planeta CS 2007 Chronic restraint or variable stresses differently affect the behavior, corticosterone secretion and body weight in rats. Physiology \& Behavior 90 29-35. (doi:10.1016/j.physbeh. 2006.08.021)

Martins JM, Banks WA \& Kastin AJ 1997 Transport of CRH from mouse brain directly affects peripheral production of $\beta$-endorphin by the spleen. American Journal of Physiology 273 E1083-E1089.
McCormick CM, Smith C \& Mathews IZ 2008 Effects of chronic social stress in adolescence on anxiety and neuroendocrine response to mild stress in male and female rats. Behavioural Brain Research 187 228-238. (doi:10.1016/j.bbr.2007.09.005)

McCormick CM, Nixon F, Thomas C, Lowie B \& Dyck J 2010 Hippocampal cell proliferation and spatial memory performance after social instability stress in adolescence in female rats. Behavioural Brain Research 208 23-29. (doi:10.1016/j.bbr.2009.11.003)

O'Keane V, Frodl T \& Dinan TG 2012 A review of atypical depression in relation to the course of depression and changes in HPA axis organization. Psychoneuroendocrinology 37 1589-1599. (doi:10.1016/ j.psyneuen.2012.03.009)

Panagiotakopoulos L \& Neigh GN 2014 Development of the HPA axis: where and when do sex differences manifest? Frontiers in Neuroendocrinology 35 285-302. (doi:10.1016/j.yfrne.2014.03.002)

Rademacher DJ, Meier SE, Shi L, Ho WS, Jarrahian A \& Hillard CJ 2008 Effects of acute and repeated restraint stress on endocannabinoid content in the amygdala, ventral striatum, and medial prefrontal cortex in mice. Neuropharmacology 54 108-116. (doi:10.1016/j.neuropharm. 2007.06.012)

Revest JM, Di Blasi F, Kitchener P, Rouge-Pont F, Desmedt A, Turiault M, Tronche F \& Piazza PV 2005 The MAPK pathway and Egr-1 mediate stress-related behavioral effects of glucocorticoids. Nature Neuroscience $\mathbf{8}$ 664-672. (doi:10.1038/nn1441)

Romeo RD 2010 Adolescence: a central event in shaping stress reactivity. Developmental Psychobiology 52 244-253. (doi:10.1002/dev.20437)

Romeo RD \& McEwen BS 2006 Stress and the adolescent brain. Annals of the New York Academy of Sciences 1094 202-214. (doi:10.1196/annals.1376.022)

Romeo RD, Bellani R, Karatsoreos IN, Chhua N, Vernov M, Conrad CD \& McEwen BS 2006 Stress history and pubertal development interact to shape hypothalamic-pituitary-adrenal axis plasticity. Endocrinology 147 1664-1674. (doi:10.1210/en.2005-1432)

Romeo RD, Kaplowitz ET, Ho A \& Franco D 2013 The influence of puberty on stress reactivity and forebrain glucocorticoid receptor levels in inbred and outbred strains of male and female mice. Psychoneuroendocrinology 38 592-596. (doi:10.1016/j.psyneuen.2012.07.019)

Silingardi D, Angelucci A, De Pasquale R, Borsotti M, Squitieri G, Brambilla R, Putignano E, Pizzorusso T \& Berardi N 2011 ERK pathway activation bidirectionally affects visual recognition memory and synaptic plasticity in the perirhinal cortex. Frontiers in Behavioral Neuroscience 5 84. (doi:10.3389/fnbeh.2011.00084)

Toledo-Rodriguez M \& Sandi C 2011 Stress during adolescence increases novelty seeking and risk-taking behavior in male and female rats. Frontiers in Behavioral Neuroscience 5 17. (doi:10.3389/fnbeh.2011.00017)

Weinberg MS, Johnson DC, Bhatt AP \& Spencer RL 2010 Medial prefrontal cortex activity can disrupt the expression of stress response habituation. Neuroscience 168 744-756. (doi:10.1016/j.neuroscience.2010.04.006)

Zippel R, Gnesutta N, Matus-Leibovitch N, Mancinelli E, Saya D, Vogel Z \& Sturani E 1997 Ras-GRF, the activator of Ras, is expressed preferentially in mature neurons of the central nervous system. Brain Research. Molecular Brain Research 48 140-144. (doi:10.1016/S0169. 328X(97)00120-4)

Received in final form 30 July 2015

Accepted 5 August 2015

Accepted Preprint published online 5 August 2015 http://joe.endocrinology-journals.org

DOI: 10.1530/JOE-15-0304
(C) 2015 Society for Endocrinology Printed in Great Britain
Published by Bioscientifica Ltd. 